\section{Периоперационное ведение}

пациентов с сопутствующим

морбидным ожирением.

Методические рекомендации

Общероссийской

общественной организации

«Федерация анестезиологов

\section{и реаниматологов»}

И.Б. Заболотских ${ }^{1}$, М.А. Анисимов², Е.С. Горобец', А.И. Грицан ${ }^{4}$, К.М. Лебединский ${ }^{5,6}$, Т.С. Мусаева', Д.Н. Проценко ${ }^{7,8}$, Н.В. Трембач ${ }^{1}$, Р.В. Шадрин ${ }^{9}$, Е.М. Шифман ${ }^{10}$, С.Л. Эпштейн ${ }^{11}$

1 ФГБОУ ВО «Кубанский государственный медицинский университет Минздрава России», Краснодар, Россия

${ }^{2}$ ФГБУ ФНКЦСМ ФМБА РоссИи, Москва, Россия

${ }^{3}$ ФГБУ «НМИЦ онкологии имени Н.Н. Блохина» Минздрава

России, Москва, Россия

${ }^{4}$ ФГБОУ ВО «Красноярский государственный медицинский университет имени профессора В.Ф. Войно-Ясенецкого»

Минздрава России, Красноярск, Россия

${ }^{5}$ ФГБОУВО «Северо-Западный государственный

медицинский университет им. И.И. Мечникова» Минздрава

России, Санкт-Петербург, Россия

${ }^{6}$ НИИ общей реаниматологии имени В.А. Неговского ФНКЦ реаниматологии и реабилитологии Минобрнауки России,

Москва, Россия

7 ФДПО ФГБОУ ВО «Российский национальный

исследовательский медицинский университет имени

Н.И. Пирогова» Минздрава России, Москва, Россия

8 ГБУз «Городская клиническая больница № 40» ДЗМ, Москва,

Россия

9 ГБУЗ «Детская краевая клиническая больница» МЗ КК,

Краснодар, Россия

10 ФУВ ГБУз МО «МОНИКИ имени М.Ф. Владимирского»,

Москва, Россия

${ }^{11}$ Клиника «ЦЭЛТ», Москва, Россия

\section{Peфepam}

По последним оценкам Всемирной организации здравоохранения, более 1 млрд человек в мире имеют избыточную массу тела. Несмотря на значительные экономические затраты по профилактике и лечению ожирения,
Perioperative management of patients with concomitant morbid obesity. Guidelines of the All-Russian public organization "Federation of Anesthesiologists and Reanimatologists"

\section{I.B. Zabolotskikh1, M.A. Anisimov², E.S. Gorobets ${ }^{3}$,} A.I. Gritsan ${ }^{4}$, K.M. Lebedinskiii, ${ }^{5,6}$, T.S. Musaeva ${ }^{1}$, D.N. Protsenko ${ }^{7,8}$, N.V. Trembach ${ }^{1}$, R.V. Shadrin ${ }^{9}$, E.M. Shifman ${ }^{10}$, S.L. Epstein ${ }^{11}$

${ }^{1}$ Kuban State Medical University, Krasnodar, Russia

2 Federal Scientific and Clinical Center of FMBA of Russia, Moscow, Russia

${ }^{3}$ N.N. Blokhin Russian Cancer Research Center, Moscow, Russia

${ }^{4}$ Voino-Yasenetsky Krasnoyarsk State Medical University, Krasnoyarsk, Russia

${ }^{5}$ North-Western State Medical University named after

I.I. Mechnikov, St. Petersburg, Russia

${ }^{6}$ Federal Research and Clinical Center for Intensive Care and Rehabilitation, Moscow, Russia

${ }^{7}$ Pirogov Russian National Research Medical University, Moscow, Russia

${ }^{8}$ City Clinical Hospital \# 40 DZM, Moscow, Russia

${ }^{9}$ Children's Regional Clinical Hospital, Krasnodar, Russia

10 Moscow Regional Research and Clinical Institute, Moscow, Russia

${ }^{11}$ CELT Clinic, Moscow, Russia

\section{Abstract}

According to the latest estimates of the World Health Organization, more than 1 billion people in the world are overweight. Despite the significant economic costs for the prevention and treatment of obesity, mortality at the age of 20-35 with morbid obesity exceeds the average statistical 12 times, mainly from cardiovascular complications. The guidelines provides principles of perioperative management of patients with obesity.

Keywords: obesity, obstructive sleep apnea, hypoventilation syndrome

凶 For correspondence: Igor B. Zabolotskikh - Dr. med. Sci., professor, head of the Department of Anesthesiology, 
летальность в возрасте 20-35 лет при патологическом ожирении превышает среднестатистическую в 12 раз, в основном от сердечно-сосудистых осложнений. В рекомендациях представлены принципы периоперационного ведения пациентов с ожирением.

Ключевые слова: ожирение, обструктивное сонное апноэ, синдром гиповентиляции

\ Для корреспонденции: Заболотских Игорь Борисович - д-р мед. наук, профессор, заведующий кафедрой анестезиологии, реаниматологии и трансфузиологии ФПК и ППС ФГБОУ ВО КубГМУ Минздрава России, Краснодар; e-mail: pobeda_zib@ mail.ru

匹 Для цитирования: И.Б. Заболотских, М.А. Анисимов, Е.С. Горобец, А.И. Грицан, К.М. Лебединский, Т.С. Мусаева, Д.Н. Проценко, Н.В. Трембач, Р.В. Шадрин, Е.М. Шифман, С.Л. Эпштейн. Периоперационное ведение пациентов с сопутствующим морбидным ожирением. Методические рекомендацииОбщероссийскойобщественнойорганизации «Федерация анестезиологов и реаниматологов». Вестник интенсивной терапии им. А.И. Салтанова. 2021;1:7-18. DOI: $10.21320 / 1818-474 X-2021-1-7-18$

๘ Поступила: 11.11 .2020

ㅁ Принята кпечати: 05.03.2021
Reanimatology and Transfusiology, Kuban State Medical University, Krasnodar, Russia; e-mail: pobeda_zib@mail.ru

¿ For citation: I.B. Zabolotskikh, M.A. Anisimov, E.S. Gorobets, A.I. Gritsan, K.M. Lebedinskii, T.S. Musaeva, D.N. Protsenko, N.V. Trembach, R.V. Shadrin, E.M. Shifman, S.L. Epstein. Perioperative management of patients with concomitant morbid obesity. Guidelines of the All-Russian public organization "Federation of Anesthesiologists and Reanimatologists". Annals of Critical Care. 2021;1:7-18. DOI: 10.21320/1818-474X-2021-1-7-18

$\uplus$ Received: 11.11.2020

G Accepted: 05.03.2021

\section{Термины и определения}

Ожирение - это хроническое обменное заболевание, которое может возникать в любом возрасте, проявляется увеличением избыточной массы тела преимущественно за счет чрезмерного накопления жировой ткани, приводя в ряде случаев к увеличению общей заболеваемости и смертности.

Обструктивное сонное апноэ - вид апноэ, характеризующийся прекращением вентиляции легких во время сна более чем на 10 с. Длительность составляет 20-30 с, в тяжелых случаях достигает 2-3 мин и занимает до 60 \% всего времени ночного сна. При систематических апноэ (обычно более 10-15 в течение часа) возникает синдром сонного апноэ с дневной сонливостью и нарушением структуры сна, жалобами на снижение работоспособности и постоянную усталость, а также ухудшением памяти и интеллекта. Различают центральное и обструктивное сонное апноэ, а также их смешанные формы.
Синдром гиповентиляции - патологическое coстояние пациента с ожирением (индекс массы тела $>30$ кг/ $\mathbf{M}^{2}$ ), когда развивается хроническая альвеолярная гиповентиляция - гиперкапническая дыхательная недостаточность, которую нельзя объяснить никакими другими причинами, кроме избыточного веса.

\section{1. Предоперационная оценка}

『 Рекомендация 1. Для диагностики ожирения и определения его степени рекомендуется рассчитывать индекс массы тела [2-5] (УДД - 1, Уур - А).

Диагноз «ожирение» устанавливается при ИМТ > 30 кг/м², «морбидное ожирение» - при ИМТ $>40$ кг/ м $^{2}$ (табл. 1). С клинической точки зрения особое внимание следует обращать на пациентов с абдоминальным типом ожирения (ИМТ > 40 кг/ $\mathrm{M}^{2}$ ), что соответствует 3-му классу по ASA. 
Таблица 1. Классификация ожирения

Table 1. Classification of obesity

\begin{tabular}{|lll|}
\hline \multicolumn{2}{|c|}{ Описание } & ИМт (кг/м²) \\
\hline Дефицит веса & $<18,5$ \\
\hline Нормальный вес & $18,5-24,9$ \\
\hline Избыточный вес & $25-29,9$ \\
\hline Ожирение & 1-я степень & $30-34,9$ \\
\cline { 2 - 3 } & 2-я степень & $35,0-39,9$ \\
\hline $\begin{array}{l}\text { Морбидное } \\
\text { ожирение }\end{array}$ & 3-я степень & $\geq 40$ \\
\cline { 2 - 3 } & 4-я степень & $\geq 50$ \\
\cline { 2 - 3 } & 5-я степень & $\geq 60$ \\
\hline
\end{tabular}

『 Рекомендация 2. У пациентов с ожирением рекомендуется определение окружности талии [6-10] (УДД - 3, Уур - В).

Абдоминальное ожирение связано с большей частотой сопутствующих заболеваний. Окружность талии более 102 см у мужчин и 88 см у женщин сопровождается увеличением риска метаболических нарушений [11].

『 Рекомендация 3. У пациентов с ожирением для скрининга обструктивного сонного апноэ (ОСА) рекомендуется применение STOP-Bang-анкеты [12-16] (УДД - 2, уУР - А).

Диагностирование обструктивного сонного апноэ (OCA) критически важно, так как его присутствие определяет повышенную чувствительность к седативным препаратам, высокую частоту гиповентиляции, гипоксемии, трудной масочной вентиляции легких и послеоперационных легочных осложнений. В большинстве случаев длительно существующий синдром OCA может вызвать компенсаторный эритроцитоз, поражение миокарда, гипоксемию, персистирующую гиперкапнию. Было найдено научное подтверждение взаимосвязи ОСА и нарушений со стороны сердечно-сосудистой системы: фибрилляции предсердий, легочной гипертензии, системной гипертензии, заболеваний коронарных артерий, инсультов, гиперкоагуляции, наступления внезапной смерти во сне $[17,18]$.

\section{STOP-Bang-анкета для скрининга обструктивного сонного апноэ}

- Храп. Вы храпите громко?

- Наблюдение. Кто-нибудь наблюдал у вас остановки дыхания во сне?
- Усталость. Часто ли вы ощущаете сонливость или усталость в дневное время?

- Артериальное давление. Имеется ли у вас артериальная гипертензия?

- Мужской пол?

- ИМТ > $35 \mathrm{\kappa г} / \mathrm{M}^{2}$ ?

- Окружность шеи $>40$ см?

- Возраст $>50$ лет?

Ответ «да» на 3 вопроса и более говорит о высоком риске ОСА.

『 Рекомендация 4. У пациентов с ожирением рекомендуется диагностика синдрома гиповентиляции с помощью анализа газов артериальной крови [19-22] (УДД - 3, уУР - В).

Синдром гиповентиляции при ожирении включает нарушение контроля дыхания во время бодрствования, увеличение напряжения $\mathrm{CO}_{2}$ в артериальной крови $\left(\mathrm{PaCO}_{2}>45\right.$ мм рт. ст.) в совокупности с ИМТ

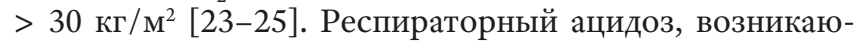
щий в ночное время, проходит в дневное [26]. Однако при продолжительном ОСА возможно развитие центрального апноэ во время бодрствования. Это отражает прогрессирующую десенсибилизацию дыхательного центра к ночной гиперкапнии.

При морбидном ожирении крайняя степень гиповентиляции приводит к синдрому Пиквика [27], который характеризуется:

- артериальной гипоксемией;

- дневной сонливостью;

- легочной гипертензией;

- гиперкапнией;

- полицитемией;

- правожелудочковой недостаточностью;

- респираторным ацидозом.

Особенности периоперационного периода при ОСА и синдроме гиповентиляции [27]:

- повышенная чувствительность к снотворным и седативным препаратам, а также опиоидам;

- вероятность трудной масочной вентиляции и трудной интубации;

- вероятность быстрой десатурации при индукции анестезии;

- вероятность гиперкапнии во время анестезии.

『 Рекомендация 5. У пациентов с ожирением рекомендуется оценка трудных дыхательных путей [28-31] (УДД - 2, уур - А).

У всех больных с патологическим ожирением следует оценить вероятность трудной масочной вентиляции и сложностей при интубации трахеи. Существуют специфичные признаки для данной категории больных: ИМТ > 26 кг $/ \mathrm{M}^{2}$ [32], окружность шеи > 40 см [33], подозрение на ОСА (храп). Должны быть продуманы заранее 
способы обеспечения проходимости верхних дыхательных путей и подготовлен запасной план действий.

『 Рекомендация 6. У пациентов с ожирением рекомендуется диагностика хронической сердечной недостаточности [34-36] (УДД-3, УУР - В).

У большинства пациентов с патологическим ожирением могут обнаруживаться симптомы сердечной недостаточности: одышка, сниженная переносимость физических нагрузок и отеки $[37,38]$. На предоперационном этапе важно произвести дифференциальную диагностику каждого из признаков и исключить или подтвердить наличие сердечной недостаточности. За объективный критерий, который определяет степень сердечной недостаточности, принимают фракцию выброса левого желудочка, измеренную при помощи эхокардиографии [39]. Однако в интерпретацию результатов может вносить вклад человеческий фактор, что следует учитывать при анализе результатов. Основным анамнестическим показателем оценки степени сердечной недостаточности является переносимость физической нагрузки. Большинство пациентов с патологическим ожирением имеют пониженную переносимость физических нагрузок, это может сопровождаться одышкой [40]. Декомпенсация диастолической дисфункции в операционный и послеоперационный период может повысить риск возникновения нарушений ритма сердца, ишемии миокарда [41].

『 Рекомендация 7. У пациентов с ожирением рекомендуется диагностика сахарного диабета [42-45] (УДД-3, уур -А).

Более чем у 90 \% пациентов с СД 2-го типа наблюдается ожирение [46], а выделяемые жировой тканью свободные жирные кислоты и цитокины нарушают чувствительность к инсулину.

Скрининг пациентов с возможным СД 2-го типа среди больных с сердечно-сосудистыми заболеваниями следует начинать с определения уровня гликированного гемоглобина и глюкозы крови натощак, если есть сомнения в постановке диагноза, применяется тест толерантности к глюкозе (IA) [47].

Гипергликемия - независимый фактор риска послеоперационных осложнений. В периоперационный период необходимо стремиться к нормогликемии. Пероральные сахароснижающие препараты необходимо отменить вечером накануне операции по причине предоперационного голодания. В период голодания перед операцией следует сокращать дозы инсулина и тщательно мониторировать уровень глюкозы. В периоперационный период из-за нарушений абсорбции подкожный путь введения инсулина не подтвердил свою эффективность. Оптимальным способом является внутривенное титрование простого инсулина $[1,26]$.
『 Рекомендация 8. У пациентов с ожирением для профилактики аспирации рекомендуется применять метоклопрамид в дозе 10 мг перорально за 1-2 ч до операции или в/в медленно за 30-40 мин и ингибиторы протонной помпы в двойной дозе (например, омепразол 40 мг) накануне вечером и за 2-4 ч до операции $[48,49]$ (УДД - 3, уУр - В).

Имеются противоречивые данные, что у пациентов с ожирением повышен риск аспирации и развития аспирационной пневмонии вследствие повышения внутрибрюшного давления, задержки эвакуации содержимого желудка, частого диагностирования грыжи пищеводного отверстия диафрагмы и гастроэзофагеального рефлюкса [1, 27]. Ингибиторы протонной помпы показаны всем пациентам с изжогой, основным симптомом гастроэзофагеального рефлюкса.

『 Рекомендация 9. У пациентов с ожирением рекомендуется профилактика тромбоэмболических осложнений [50-53] (УДД - 2, УУР - А).

Риск тромбообразования глубоких вен у пациентов с ожирением, перенесших оперативное вмешательство, приблизительно вдвое выше, чем у обычных людей. Возрастание риска тромбоэмболий у тучных пациентов - последствие полицитемии, иммобилизации и увеличения внутрибрюшного давления, что приводит к венозному застою.

Предоперационное выявление тромбоза глубоких вен нижних конечностей должно сопровождаться ранней профилактикой венозных тромбоэмболических осложнений: компрессионный трикотаж, ранняя активизация, низкомолекулярные гепарины. При выявлении гиперкоагуляции профилактику венозных тромбоэмболических осложнений следует начинать до оперативного вмешательства. При увеличении показателей $\mathrm{D}$-димера, даже при отсутствии данных, свидетельствующих о наличии тромбоза глубоких вен нижних конечностей, необходимо рассмотреть возможность назначения «лечебных» доз низкомолекулярных гепаринов.

\section{2. Интраоперационное ведение}

『 Рекомендация 10. Перед индукцией рекомендуется укладывать пациента в горизонтальное положение с поднятым головным концом или в обратное положение Тренделенбурга для увеличения ФОЕ и улучшения оксигенации [54-57] (УДД - 2, УУР A).

Необходимо уложить пациента так, чтобы линия, соединяющая грудину и ухо пациента, расположилась горизонтально (так называемая позиция для ларинго- 
скопии с поднятым краниальным концом - HELP-позиция). Это достигается подкладыванием под плечи и спину больного подушек, полотенец и одеял. Именно это положение обеспечивает лучшие условия для интубации трахеи, упрощает масочную вентиляцию и ларингоскопию [58].

『 Рекомендация 11. Для анестезии у пациентов с ожирением рекомендуется пропофол или современные ингаляционные анестетики - севофлуран и десфлуран [59-64] (УДД-3, УУР - В).

У пациентов с ожирением для быстрого восстановления (т. е. почти всегда) следует использовать анестетики с низкой растворимостью в крови и низкой липофильностью [1, 41]. Применение анестетиков с высокой липофильностью у пациентов с ожирением приводит к замедленному пробуждению [65]. Севофлуран и десфлуран - препараты выбора у тучных пациентов. Десфлуран приобрел популярность в бариатрической хирургии в связи с особо благоприятным профилем пробуждения. Анестезия десфлураном может оказаться более управляемой по сравнению с наркозом севофлураном, поскольку десфлуран практически не кумулирует независимо от продолжительности наркоза [66]. В современной практике анестезиолога пропофол стал препаратом выбора для вводного наркоза у пациентов с ожирением [41]. Оптимальное дозирование необходимо проводить методом титрования. Следует помнить, что у пациентов с ОСА применение пропофола и бензодиазепинов для седации связано с риском гипоксемии. Масочный наркоз десфлураном непригоден для индукции из-за едкого, раздражающего запаха.

『 Рекомендация 12. У пациентов с ожирением рекомендуется коррекция дозы препаратов для анестезии [67-69] (УДД - 3, уУР - В).

У пациентов с ожирением для расчета дозы используют тощий вес тела и идеальный вес тела (табл. 2). Метод расчета зависит от препарата (табл. 3).

『 Рекомендация 13. У пациентов с ожирением во время преоксигенации и индукции анестезии рекомендуется использовать постоянное положительное давление в дыхательных путях и фракцию вдыхаемого кислорода не более 80 \% под контролем $\mathrm{SpO}_{2} \geq 95 \%$, а у пациентов с «трудными» дыхательными путями $\mathrm{FiO}_{2}$ должно быть 100 \% [70-73] (УДД - 2, Уур - А).
Таблица 2. Формулы расчета идеального и тощего веса тела

Table 2. Ideal Body Weight and Lean Body Weight Formulas

\begin{tabular}{|c|c|}
\hline $\begin{array}{l}\text { Расчетный } \\
\text { показатель }\end{array}$ & Формула \\
\hline $\begin{array}{l}\text { Индекс массы } \\
\text { тела (кг/м²) }\end{array}$ & Общий вес тела / рост² \\
\hline $\begin{array}{l}\text { Идеальный вес } \\
\text { тела } \\
\text { (кг) }\end{array}$ & $\begin{array}{l}\text { Рост (см)-V, } \\
V=100 \text { для взрослых мужчин, } \\
V=105 \text { для взрослых женщин }\end{array}$ \\
\hline $\begin{array}{l}\text { Тощий вес тела } \\
\text { (ТВТ) }\end{array}$ & 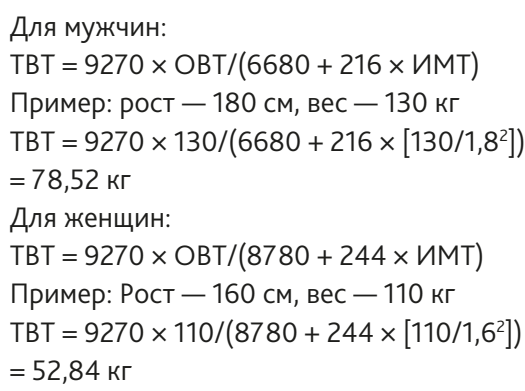 \\
\hline
\end{tabular}

Таблица 3. Принципы расчета доз препаратов для анестезии при ожирении

Table 3. The principles of calculating doses of drugs for anesthesia for obesity

\begin{tabular}{|ll|}
\hline \multicolumn{1}{|c|}{ Препараты } & \multicolumn{1}{c|}{ Вес для расчета дозы } \\
\hline Пропофол & $\begin{array}{l}\text { Индукция - ТВТ } \\
\text { Поддержание - ОВТ }\end{array}$ \\
\hline Тиопентал натрия & $\begin{array}{l}\text { Индукция - ТВТ } \\
\text { Поддержание - ОВТ }\end{array}$ \\
\hline Мидазолам & Индукция - ОВТ \\
\hline Дексмедетомидин & Поддержание - ИВТ \\
\hline Рокурония бромид & ИВТ или ТВТ \\
\hline Атракурия бензилат & ОВТ \\
\hline Цисатракурия бензилат & ИВТ или ТВТ \\
\hline Суксаметония хлорид & ОВТ \\
\hline Неостигмина метилсульфат & ОВТ \\
\hline Фентанил & ТВТ \\
\hline Морфин & ИВТ \\
\hline Парацетамол & ИВТ \\
\hline ИВТ - расчет препарата на идеальный вес тела;
\end{tabular}


『 Рекомендация 14. У пациентов с ожирением рекомендуется применять неинвазивную вентиляцию легких (НИВЛ) или высокопоточную оксигенацию (ВПО) вместо стандартной преоксигенации в течение индукции анестезии $[74,75]$ (УДД - 2, УУР - В).

Как неинвазивная вентиляция легких (НИВЛ), так и высокопоточная оксигенация (ВПО) обеспечивают лучшую оксигенацию по сравнению со стандартной преоксигенацией у пациентов с ожирением. Тем не менее конечно-экспираторное давление, генерируемое ВПО (не более 5 см вод. ст.), может быть недостаточным для профилактики ателектазирования у пациентов с избыточной массой тела [76]. По данным литературы, при применении НИВЛ частота умеренной гипоксемии ниже, чем при применении ВПО при сопоставимой частоте осложнений, однако переносимость ВПО лучше.

『 Рекомендация 15. У пациентов с ожирением рекомендуется применение малых дыхательных объемов (менее 10 мл/кг ДМТ) и положительного конечно-экспираторного давления не менее 10 см вод. ст. во время искусственной вентиляции легких [77-82] (УДД - 2, УУР - В).

『 Рекомендация 16. У пациентов с ожирением уровень конечно-экспираторного давления выше 10 см вод. ст. рекомендуется сочетать с профилактическим применением маневра открытия альвеол [82-89] (УДД - 3, УУР - В).

Применение положительного конечно-экспираторного давления позволяет улучшить биомеханику дыхания, препятствовать развитию ателектазов [85-87]. Маневр открытия альвеол обеспечивает мобилизацию альвеол, коллапс которых уже произошел в течение индукции анестезии, при механическом воздействии на легкие, после дисконнекции дыхательного контура [82-88]. Сочетание РEЕР и маневра открытия альвеол снижает частоту гипоксемии, однако рекомендуемая стратегия должна выполняться с осторожностью, поскольку сопряжена с увеличением риска гипотензии и брадикардии [89]. Тем не менее профилактическое применение маневра открытия альвеол связано с менышей частотой послеоперационных легочных осложнений по сравнению с маневром, выполненным по поводу уже возникшей гипоксемии [90].

『 Рекомендация 17. У пациентов с ожирением рекомендуется применять маневр открытия альвеол методом пошагового увеличения конечно-экспираторного давления с неизменным движущим давлением $[90,91]$ (УДД - 3, УУР - В).
Обсервационное исследование выявило снижение частоты послеоперационных легочных осложнений у пациентов с ожирением при применении маневра раскрытия альвеол с помощью наркозного аппарата по сравнению с маневром, выполненным вручную мешком аппарата [90]. Метод пошагового увеличения конечно-экспираторного давления с неизменным движущим давлением әффективно улучшает биомеханику дыхания у пациентов, сохраняя гемодинамику стабильной [91].

『 Рекомендация 18. У пациентов с ожирением рекомендуется применение регионарных и нейроаксиальных блокад [92-97] (УДД - 3, уУР - А).

\section{3. Ведение в послеоперационном периоде}

『 Рекомендация 19. При наличии остаточной нейромышечной блокады рекомендовано использовать декураризацию под объективным контролем нервно-мышечной передачи с целевым значением TOF > 0,9 (90 \%) [98-103] (УдД - 2, уУР - В).

Спонтанное восстановление НМП происходит медленно и непредсказуемо, зависит от сопутствующей терапии, типа анестезии и индивидуальных особенностей пациента.

Клинические признаки восстановления НМП ненадежны, особенно малоинформативны показатели спирометрии. Единственный метод объективной оценки состояния НМП, пригодный для рутинной анестезиологической работы, в особенности для принятия решения об экстубации, это мониторинг ответа на ТOF-стимуляцию.

Ингибиторы холинэстеразы остаются основным средством устранения остаточного НМБ, но их эффективность ограничена целым рядом условий, а действие проявляется медленно. При глубокой миоплегии ингибиторы холинэстеразы в принципе не способны восстанавливать НМП. Кроме того, они обладают множеством побочных реакций, а при передозировке могут сами вызывать дыхательную недостаточность из-за избыточного холиномиметического действия на нервно-мышечные синапсы.

Сугаммадекс способен в течение 1-4 мин полностью прекращать даже глубокий НМБ, вызванный аминостероидным миорелаксантом - рокурония бромидом.

『 Рекомендация 20. Экстубацию пациентов с ожирением рекомендуется проводить после восстановления нервно-мышечной передачи в положении сидя или полусидя, сопровождая послеоперационный период методами профилактики послеоперационных легочных осложнений [104-109] (УДД - 3, УУР - А). 
Таблица 4. Рекомендации по дозированию препаратов для тромбопрофилактики

Table 4. Dosing recommendations for thromboprophylaxis

\begin{tabular}{|c|c|c|c|c|}
\hline \multirow{2}{*}{ Препарат } & \multicolumn{4}{|c|}{ Масса тела, кг } \\
\hline & $<50$ & $50-100$ & $100-150$ & $>150$ \\
\hline Эноксапарин натрия & 20 мг 1 р./день & 40 мг 1 р./день & 40 мг 2 р./день & 60 мг 2 р./день \\
\hline Далтепарин натрия & 2500 МЕ 1 р./день & 5000 МЕ 1 р./день & 5000 ME 2 р./день & 7500 МЕ 2 р./день \\
\hline
\end{tabular}

『 Рекомендация 21. У пациентов с ожирением рекомендуется применение мультимодальной послеоперационной анальгезии [110-113] (УДД - 3, уур - В).

После обширных оперативных вмешательств рекомендуется обезболивание путем сочетания опиатов (фентанил) с нестероидными противовоспалительными препаратами и/или парацетамолом [1]. Парацетамол возможно применять только при отсутствии у пациента явлений печеночной дисфункции. Дозы: парацетамол (Перфалган) 4-5 г/сут 4-5 дней, кеторолак 90 мг/сут (в/м) (или лорноксикам 32 мг/сут) 3-4 дня [114].

『 Рекомендация 22. У пациентов с ожирением для послеоперационного обезболивания рекомендуется применение продленных регионарных и нейроаксиальных блокад [115-122] (УДД - 3, уур - А).

『 Рекомендация 23. У пациентов с ожирением рекомендуется проведение послеоперационной тромбопрофилактики [123-128] (УДД - 2, УУР - А).

Вероятность тромбоза глубоких вен и тромбоэмболии легочной артерии у тучных пациентов значительно увеличивается. Настоятельно требуются ранняя активизация и использование антикоагулянтов. Это могут быть низкомолекулярные гепарины либо новые перо- ральные препараты (равароксабан, дабигатран и т. п.). В периоперационный период целесообразна эластическая компрессия нижних конечностей. Для этой цели следует использовать эластические чулки. Применение эластических бинтов нецелесообразно, а иногда и опасно из-за невозможности обеспечить равномерную компрессию необходимой силы. С появлением новых пероральных антикоагулянтов (ривароксабан, дабигатрана этексилат и т. д.) при условии применения этих препаратов до операции к их приему следует вернуться как можно раньше, т. е. когда риск послеоперационного кровотечения представляется хирургу минимальным.

Профилактика тромбозов в ранний послеоперационный период [1]:

- эластичная компрессия нижних конечностей чулками (применение эластических бинтов нецелесообразно, а иногда и опасно из-за невозможности создать равномерную компрессию должной силы);

- ранняя послеоперационная активизация;

- применение низкомолекулярных гепаринов (рассчитывается на общую массу тела).

Однако в настоящее время нет достаточной доказательной базы о преимуществе какого-либо препарата или схемы его применения [114]. Одновременно с этим следует учитывать риск развития послеоперационных кровотечений на фоне терапии гепаринами. С одинаковой степенью эффективности применяются нефракционированный гепарин по 5000-7500 МЕ подкожно каждые 8 ч, внутривенная инфузия гепарина $400 \mathrm{ME} /$, подкож-

Таблица 5. Критерии оценки качества медицинской помощи

Table 5. Criteria for assessing the quality of medical care

\begin{tabular}{|c|c|c|c|}
\hline № & Критерии качества & $\begin{array}{l}\text { Уровень достоверно- } \\
\text { сти доказательств }\end{array}$ & $\begin{array}{c}\text { Уровень убедительности } \\
\text { рекомендаций }\end{array}$ \\
\hline 1 & Определен индекс массы тела & 1 & A \\
\hline 2 & Оценен риск трудных дыхательных путей & 2 & A \\
\hline 3 & $\begin{array}{l}\text { Перед индукцией пациент находился в горизонтальном положении с подня- } \\
\text { тым головным концом или в обратном положении Тренделенбурга }\end{array}$ & 2 & A \\
\hline 4 & $\begin{array}{l}\text { Экстубация пациента проведена после восстановления нервно-мышечной } \\
\text { передачи в положении сидя или полусидя }\end{array}$ & 3 & A \\
\hline 5 & Произведена тромбопрофилактика & 2 & $A$ \\
\hline
\end{tabular}


ное введение надропарина кальция 0,3-0,6 мл через 12 ч, эноксапарина натрия 30-40 мг 1-2 раза в сутки [129]. Следует начинать антикоагулянтную терапию как можно раньше после оперативного вмешательства (табл. 4).

\section{4. Дополнительная информация}

Конфликт интересов. Авторы заявляют об отсутствии конфликта интересов.

Вклад авторов. Заболотских И.Б., Анисимов М.А., Горобец Е.С., Грицан А.И., Лебединский К.М., Мусаева Т.С., Проценко Д.Н., Трембач Н.В., Шадрин Р.В., Шифман Е.М.,
Эпштейн С.Л. - разработка концепции статьи, получение и анализ фактических данных, написание и редактирование текста статьи, проверка и утверждение текста статьи.

ORCID авторов
Заболотских И.Б. - 0000-0002-3623-2546
Анисимов М.А. - 0000-0002-4484-6716
Горобец Е.С. - 0000-0002-9311-2153
Грицан А.И. - 0000-0002-0500-2887
Лебединский К.М. - 0000-0002-5752-4812
Мусаева Т.С. - 0000-0001-9285-852X
Проценко Д.Н. - 0000-0002-5166-3280
Трембач Н.В. - 0000-0002-0061-0496
Шадрин Р.В. - 0000-0002-0249-6615
Шифман Е.М. - 0000-0002-6113-8498

\section{Литература/References}

[1] Bellami M., Struys M. Anesthesia for the Overweight and Obese Patient. 2007; 234 p. DOI: 10.1093/med/9780199233953.001.0001

[2] Sung Y.A., Oh J.Y., Lee H. Comparison of the body adiposity index to body mass index in Korean women. Yonsei Med J. 2014; 55(4): 1028-35. DOI: 10.3349/ymj.2014.55.4.1028

[3] Habib S. S. Body mass index and body fat percentage in assessment of obesity prevalence in saudi adults. Biomed Environ Sci. 2013; 26(2): 94-9. DOI: 10.7717/peerj.3238/supp-1

[4] Lin X.J., Wang C.P., Liu X.D., et al. Body mass index and risk of gastric cancer: a meta-analysis. Jpn J Clin Oncol. 2014; 44(9): 783-91. DOI: 10.1093/jjco/hyu082

[5] Javed A., Jumean M., Murad M.H., et al. Diagnostic performance of body mass index to identify obesity as defined by body adiposity in children and adolescents: a systematic review and meta-analysis. Pediatr Obes. 2015; 10(3): 234-44. DOI: 10.1111/ijpo.242

[6] Cerhan J.R., Moore S.C., Jacobs E.J., et al. A pooled analysis of waist circumference and mortality in 650,000 adults. Mayo Clin Proc. 2014; 89(3): 335-45. DOI: 10.1016/j.mayocp.2013.11.011

[7] Florath I., Brandt S., Weck M.N., et al. Evidence of inappropriate cardiovascular risk assessment in middle-age women based on recommended cut-points for waist circumference. Nutr Metab Cardiovasc Dis. 2014; 24(10): 1112-9. DOI: 10.1016/j.numecd.2014.04.005

[8] Tanamas S.K., Shaw J.E., Backholer K., et al. Twelve-year weight change, waist circumference change and incident obesity: the Australian diabetes, obesity and lifestyle study. Obesity (Silver Spring). 2014; 22(6): 1538-45. DOI: 10.1002/oby.20704

[9] Nunes C.N., Minicucci M.F., Farah E., et al. Impact of different obesity assessment methods after acute coronary syndromes. Arq Bras Cardiol. 2014; 103(1): 19-24. doi.org/10.5935/abc.20140073

[10] Zhou H.C., Lai Y.X., Shan Z.Y., et al. Effectiveness of different waist circumference cut-off values in predicting metabolic syndrome prevalence and risk factors in adults in China. Biomed Environ Sci. 2014; 27(5): 325-34. DOI: 10.1161/circulationaha.111.065904

[11] Labib M. The investigation and management of obesity. Clin Pathol. 2003; 56: 17-25. DOI: 10.1136/jcp.56.1.17

[12] Nagappa M., Liao P., Wong J., et al. Validation of the STOPBang Questionnaire as a Screening Tool for Obstructive Sleep Apnea among Different Populations: A Systematic Review and Meta-Analysis. PLoS One. 2015; 10(12): e0143697. DOI: 10.1371/journal.p one.0143697

[13] Khanna A.K., Sessler D.I., Sun Z., et al. Using the STOP-BANG questionnaire to predict hypoxaemia in patients recovering from noncardiac surgery: a prospective cohort analysis. Br J Anaesth. 2016; 116(5): 632-40. DOI: 10.1371/journal.pone.0143697

[14] Chudeau N., Raveau T., Carlier L., et al. The STOP-BANG questionnaire and the risk of perioperative respiratory complications in urgent surgery patients: A prospective, observational study. Anaesth Crit Care Pain Med. 2016; 35(5): 347-353. DOI: 10.1016/j. accpm.2016.01.006

[15] Corso R.M., Petrini F., Buccioli M., et al. Clinical utility of preoperative screening with STOP-Bang questionnaire in elective surgery. Minerva Anestesiol. 2014; 80(8): 877-84. DOI: 10.23736/s0375-9393.19.14308-8

[16] Chung F., Yegneswaran B., Liao P. STOP questionnaire: a tool to screen patients for obstructive sleep apnea. Anesthesiology. 2008; 108: 812-821. DOI: 10.1097/sa.0b013e31818bcea2

[17] Bradley T.D., Floras J.S. Obstructive sleep apnea and its cardiovascular consequences. Larnet. 2009; 373: 82-93. DOI: 10.1201/b15277

[18] Somers V.K., White D.P., Amin R. Sleep apnea and cardiovascular disease. Circulation. 2008; 118: 1080-1111. DOI: 10.1016/b978-1-4377-0398-6.00079-2

[19] Kaw R., Bhateja P., Paz Y., Mar H., et al. Postoperative Complications in Patients with Unrecognized Obesity Hypoventilation Syndrome Undergoing Elective Noncardiac Surgery. Chest. 2016; 149(1): 84 91. DOI: $10.1378 /$ chest.14-3216

[20] Fernandez A.Z. Jr., Demaria E.J., Tichansky D.S., et al. Multivariate analysis of risk factors for death following gastric bypass for treatment of morbid obesity. Ann Surg. 2004; 239(5): 698-702. DOI: 10.1097/01.sla.0000124295.41578.ab

[21] Lumachi F., Marzano B., Fanti G., et al. Hypoxemia and hypoventilation syndrome improvement after laparoscopic bariatric surgery 
in patients with morbid obesity. In: Vivo. 2010; 24(3): 329-31. DOI: 10.1021/acs.jproteome.8b00144.s001

[22] Raveendran R., Wong J., Singh M., et al. Obesity hypoventilation syndrome, sleep apnea, overlap syndrome: perioperative management to prevent complications. Curr Opin Anaesthesiol. 2017; 30(1): 146-155. DOI: 10.1097/aco.0000000000000421

[23] Davis G., Patel J.A., Gagne D.J. Pulmonary considerations in obesity and bariatric surgical patient. Med Clin N Am. 2007; 91: 433-442. DOI: 10.1016/j.mcna.2007.02.001

[24] Mokhlesi B., Tulaimat A. Recent advances in obesity hypoventilation syndrome. 2007; 132: 1322-1336. DOI: 10.1378/chest.07-0027

[25] Crummy F., Piper A.J., Naughton M.T. Obesity and the lung. Thorax. 2008; 63: 738-746. DOI: 10.1136/thx.2007.086843

[26] Adams J.P., Murphy P.G. Obesity in anaesthesia and intensive care. Br J Anaesth. 2000; 85: 91-108. DOI: 10.1093/bja/85.1.91

[27] Hines R.L., Marschall K.E. Anesthesia and Co-Existing Disease. 2008; 195 p. DOI: 10.1016/b978-1-4160-3998-3.10003-x

[28] Lavi R., Segal D., Ziser A. Predicting difficult airways using the intubation difficulty scale: a study comparing obese and non-obese patients. J Clin Anesth. 2009; 21(4): 264-7. DOI: 10.1016/j.jclinane.2008.08.021

[29] Williamson J.A., Webb R.K., Szekely S., et al. The Australian Incident Monitoring Study. Difficult intubation: an analysis of 2000 incident reports. Anaesth Intensive Care. 1993; 21(5): 602-7. DOI: $10.1177 / 0310057 \times 9302100518$

[30] Lima Filho J.A., Ganem E.M., de Cerqueira B.G. Reevaluation of the airways of obese patients undergone bariatric surgery after reduction in body mass index. Rev Bras Anestesiol. 2011; 61(1): 31-40. DOI: 10.1016/s0034-7094(11)70004-6

[31] Tatlipınar A., Kınal E. Links and risks associated with adenotonsillectomy and obesity. Pediatric Health Med Ther. 2015; 6: 123-127. DOI: 10.2147/phmt.s66730

[32] Langeron O., Masso E., Huraux C., et al. Prediction of difficult mask ventilation. Anesthesiology; 92: 1229-1236. DOI: 10.1097/00000542-200005000-00009

[33] Cattano D., Katsiampoura A., Corso R.M., et al. Predictive factors for difficult mask ventilation in the obese surgical population. F1000Res. 2014; 3: 239. DOI: 10.12688/f1000research.5471.1

[34] Bozkurt B., Aguilar D., Deswal A., et al. Contributory Risk and Management of Comorbidities of Hypertension, Obesity, Diabetes Mellitus, Hyperlipidemia, and Metabolic Syndrome in Chronic Heart Failure: A Scientific Statement From the American Heart Association. Circulation. 2016; 134(23): 535-578. DOI: 10.1161/cir.0000000000000450

[35] Kenchaiah S., Evans J.C., Levy D., et al. Obesity and the risk of heart failure. N Engl J Med. 2002; 347(5): 305-13. DOI: 10.1056/nejmoa020245

[36] Nagarajan V., Cauthen C.A., Starling R.C., et al. Prognosis of morbid obesity patients with advanced heart failure. Congest Heart Fail. 2013; 19(4): 160-4. DOI: 10.1111/chf.12038

[37] Alpert M. A. Obesity cardiomyopathy: pathophysiology and evolution of the clinical syndrome. Am J Med Sci. 2001; 321: 225-236. DOI: 10.1097/00000441-200104000-00003

[38] Poirier P., Giles T.D., Bray G.A., et al. Obesity and cardiovascular disease: pathophysiology, evaluation, and effect of weight loss: an update of the 1997 American Heart Association scientific statement on obesity and heart disease from the Obesity Committee of the Council on Nutrition, Physical Activity, and Metabolism. Circulation. 2006; 113: 898-918. DOI: 10.1161/circulationaha.106.171016

[39] Paulus W.J., Tschope C., Sanderson J.E., et al. How to diagnose diastolic heart failure: a consensus statement on the diagnosis of heart failure with normal left ventricular ejection fraction by the Heart Failure and Echocardiography Associations of the European Society of Cardiology. Eur Heart J. 2007; 28(20): 2539-2550. DOI: 10.1093/eurheartj/ehm380

[40] Национальные рекомендации ОССН, РКО и РНМОТ по диагностике и лечению XCH (четвертый пересмотр) 2012 г. Сердечная недостаточность. 2013; 14(7): 35. DOI: 10.18087/ cardio.2475 [Natsionalnyye rekomendatsii OSSN. RKO i RNMOT po diagnostike i lecheniyu KhSN (chetvertyy peresmotr) 2012 g. Serdechnaya nedostatochnost. 2013; 14(7): 35. (In Russ)]

[41] Morbid obesity. Perioperative management. Alvarez A., et al., eds. 2010; 246 p. DOI: 10.19052/ruls.vol1.iss77.6

[42] Crujeiras A.B., Díaz-Lagares A., Carreira M.C., et al. Oxidative stress associated to dysfunctional adipose tissue: a potential link between obesity, type 2 diabetes mellitus and breast cancer. Free Radic Res. 2013; 47(4): 243-56. DOI: 10.3109/10715762.2013.772604

[43] Frank G.R., Fox J., Candela N., et al. Severe obesity and diabetes insipidus in a patient with PCSK1 deficiency. Mol Genet Metab. 2013; 110(1-2): 191-4. DOI: 10.1016/j.ymgme.2013.04.005

[44] Fronczyk A., Molęda P., Safranow K., et al. Increased concentration of $C$-reactive protein in obese patients with type 2 diabetes is associated with obesity and presence of diabetes but not with macrovascular and microvascular complications or glycemic control. Inflammation. 2014; 37(2): 349-57. DOI: 10.1007/s10753-013-9746-4

[45] Lim R.B., Chen C., Naidoo N., et al. Anthropometrics indices of obesity, and all-cause and cardiovascular disease-related mortality, in an Asian cohort with type 2 diabetes mellitus. Diabetes Metab. 2015; 41(4): 291-300. DOI: 10.1016/j.diabet.2014.12.003

[46] Hossain P., Kawar B., El Nahas M. Obesity and diabetes in the developing world: a growing challenge. N Engl J Med. 2007; 356: 213-215. doi.org/10.1056/nejmp068177

[47] Рекомендации по диабету, предиабету и сердечно-сосудистым заболеваниям. EASD/ESC 2013 Российский кардиологический журнал. 2014; 3(107): 7-61. DOI: 10.1016/j.rec.2020.04.007 [Rekomendatsii po diabetu. prediabetu i serdechno-sosudistym zabolevaniyam. EASD/ESC 2013 Rossiyskiy kardiologicheskiy zhurnal. 2014; 3(107): 7-61. (In Russ)]

[48] Mahajan V., HashmiJ., Singh R., et al. Comparative evaluation of gastric $\mathrm{pH}$ and volume in morbidly obese and lean patients undergoing elective surgery and effect of aspiration prophylaxis. J Clin Anesth. 2015; 27(5): 396-400. DOI: 10.1016/j.jclinane.2015.03.004

[49] Cook-Sather S.D., Gallagher P.R., Kruge L.E., et al. Overweight/obesity and gastric fluid characteristics in pediatric day surgery: implications for fasting guidelines and pulmonary aspiration risk. Anesth Analg. 2009; 109(3): 727-36. DOI: 10.1213/ane.0b013e3181b085ff. doi.org/10.1213/ ane.0b013e3181b085ff

[50] Wang T.F., Milligan P.E., Wong C.A., et al. Efficacy and safety of high-dose thromboprophylaxis in morbidly obese inpatients. Thromb Haemost. 2014; 111(1): 88-93. DOI: 10.1160/th13-01-0042 
[51] Steele K.E., Canner J., Prokopowicz G., et al. The EFFORT trial: Preoperative enoxaparin versus postoperative fondaparinux for thromboprophylaxis in bariatric surgical patients: a randomized double-blind pilot trial. Surg Obes Relat Dis. 2015; 11(3): 672-83. DOI: 10.1016/j.soard.2014.10.003

[52] Ho K.M., Bham E., Pavey W. Incidence of Venous Thromboembolism and Benefits and Risks of Thromboprophylaxis After Cardiac Surgery: A Systematic Review and Meta-Analysis. J Am Heart Assoc. 2015; 4(10): e002652. DOI: 10.1161/jaha.115.002652

[53] Steib A., Degirmenci S.E., Junke E., et al. Once versus twice daily injection of enoxaparin for thromboprophylaxis in bariatric surgery: effects on antifactor $\mathrm{Xa}$ activity and procoagulant microparticles. A randomized controlled study. Surg Obes Relat Dis. 2016; 12(3): 613-621. DOI: 10.1016/j.soard.2015.08.505

[54] Dixon B.J., Dixon J.B., Carden J.R., et al. Preoxygenation is more effective in the 25 degrees head-up position than in the supine position in severely obese patients: a randomized controlled study. Anesthesiology. 2005; 102(6): 1110-5. DOI: 10.1097/00000542-200506000-00009

[55] Lane S., Saunders D., Schofield A., et al. A prospective, randomised controlled trial comparing the efficacy of pre-oxygenation in the 20 degrees head-up vs supine position. Anaesthesia. 2005; 60(11): 1064-7. DOI: 10.1111/j.1365-2044.2005.04374.x

[56] Rao S.L., Kunselman A.R., Schuler H.G., Des Harnais S. Laryngoscopy and tracheal intubation in the head-elevated position in obese patients: a randomized, controlled, equivalence trial. Anesth Analg. 2008; 107(6): 1912-8. DOI: 10.1213/ane.0b013e31818556ed

[57] Valenza F., Vagginelli F., Tiby A., et al. Effects of the beach chair position, positive end-expiratory pressure, and pneumoperitoneum on respiratory function in morbidly obese patients during anesthesia and paralysis Anesthesiology. 2007; 107(5): 725-32. DOI: 10.1097/01.anes.0000287026.61782.a6

[58] Brodsky J.B., Lemmens H.J., Brock-Utne J.G., et al. Morbid obesity and tracheal intubation. Anesth Analg. 2002; 94: 3732-3736. DOI: 10.1097/00000539-200203000-00047

[59] Cortínez L.I., Gambús P., Trocóniz I.F., et al. Obesity does not influence the onset and offset of sevoflurane effect as measured by the hysteresis between sevoflurane concentration and bispectral index. Anesth Analg. 2011; 113(1): 70-6. DOI: 10.1213/ane.0b013e31821f105c

[60] Pal D., Walton M.E., Lipinski W.J., et al. Determination of minimum alveolar concentration for isoflurane and sevoflurane in a rodent model of human mebolic syndrome. Anesth Analg. 2012 Feb; 114(2): 297-302. doi.org/10.1213/ane.0b013e31823ede22

[61] Gaszyński T., Wieczorek A. A comparison of BIS recordings during propofol-based total intravenous anaesthesia and sevoflurane-based inhalational anaesthesia in obese patients. Anaesth Intensive Ther. 2016; 48(4): 239-247. DOI: 10.1177/0310057x1804600509

[62] McKay R.E., Malhotra A., Cakmakkaya O.S., et al. Effect of increased body mass index and anaesthetic duration on recovery of protective airway reflexes after sevoflurane vs desflurane. $\mathrm{Br} J$ Anaesth. 2010 Feb; 104(2): 175-82. DOI: 10.1093/bja/aep374

[63] El-Baraky I.A., Abbassi M.M., Marei T.A., et al. Obesity Does Not Affect Propofol Pharmacokinetics During Hypothermic Cardiopul- monary Bypass. J Cardiothorac Vasc Anesth. 2016; 30(4): 876-83. DOI: 10.1053/j.jvca.2016.02.003

[64] Tachibana N., Niiyama Y., Yamakage M. Evaluation of bias in predicted and measured propofol concentrations during target-controlled infusions in obese Japanese patients: an open-label comparative study. Eur J Anaesthesiol. 2014 Dec; 31(12): 701-7. DOI: 10.1097/eja.0000000000000154

[65] Juvin P., Vadam C., Malek L., et al. Postoperative recovery after desflurane, propofol, or isoflurane anesthesia among morbidly obese patients: a prospective, randomized study. Anesth Analg. 2000; 91: 714-719. DOI: 10.1097/00000539-200009000-00041

[66] Strum E.M., Szenohradszki J., Kaufman W.A., et al. Emergence and recovery characteristics of desflurane versus sevoflurane in morbidly obese adult surgical patients: a prospective, randomized study. Anesth Analg. 2004; 99: 1848-1853. DOI: 10.1213/01.ane.0000136472.01079.95

[67] Baerdemaeker L.E., Struys M.M. Optimization of desflurane administration in morbidly obese patients. $\mathrm{Br} J$ Anesth. 2003; 91(5): 638-650. DOI: 10.1093/bja/aeg236

[68] La Colla L., Albertin A., La Colla G., et al. Predictive performance of the 'Minto' remifentanil pharmacokinetic parameter set in morbidly obese patients ensuing from a new method for calculating lean body mass. Clin Pharmacokinet. 2010; 49(2): 131-9. DOI: 10.2165/11317690-000000000-00000

[69] Shibutani K., Inchiosa M.A. Jr., Sawada K., et al. Pharmacokinetic mass of fentanyl for postoperative analgesia in lean and obese patients. $\mathrm{Br} J$ Anaesth. 2005; 95(3): 377-83. DOI: 10.1097/00000539-199902001-00378

[70] Harbut P., Gozdzik W., Stjernfält E., et al. Continuous positive airway pressure/pressure support pre-oxygenation of morbidly obese patients. Acta Anaesthesiol Scand. 2014; 58(6): 675-80. DOI: 10.1111/aas.12317

[71] Cressey D.M., Berthoud M.C., Reilly C.S. Effectiveness of continuous positive airway pressure to enhance pre-oxygenation in morbidly obese women. Anaesthesia. 2001 Jul; 56(7): 680-4. DOI: 10.1046/j.1365-2044.2001.01374-3.x

[72] Delay J.M., Sebbane M., Jung B., et al. The effectiveness of noninvasive positive pressure ventilation to enhance preoxygenation in morbidly obese patients: a randomized controlled study. Anesth Analg. 2008; 107(5): 1707-13. DOI: 10.1213/ane.0b013e318183909b

[73] Rajan S., Joseph N., Tosh P., et al. Effects of Preoxygenation with Tidal Volume Breathing Followed by Apneic Oxygenation with and without Continuous Positive Airway Pressure on Duration of Safe Apnea Time and Arterial Blood Gases. Anesth Essays Res. 2018; 12(1): 229-233. DOI: 10.4103/aer.aer_219_17

[74] Wong D.T., Dallaire A., Singh K.P., et al. High-Flow Nasal Oxygen Improves Safe Apnea Time in Morbidly Obese Patients Undergoing General Anesthesia: A Randomized Controlled Trial. Anesth Analg. 2019; 129(4): 1130-1136. DOI: 10.1213/ane.0000000000003966

[75] Stéphan F., Bérard L., Rézaiguia-Delclaux S. High-Flow Nasal Cannula Therapy Versus Intermittent Noninvasive Ventilation in Obese Subjects After Cardiothoracic Surgery. Respir Care. 2017; 62(9): 1193-1202. DOI: 10.4187/respcare.05473 
[76] Groves N., Tobin A. High flow nasal oxygen generates positive airway pressure in adult volunteers. Aust Crit Care. 2007; 20: 126131. DOI: 10.1016/j.aucc.2007.08.001

[77] Anzueto A., Frutos-Vivar F., Esteban A., et al. Influence of body mass index on outcome of the mechanically ventilated patients. Thorax. 2011; 66(1): 66-73. DOI: 10.1136/thx.2010.145086

[78] Ortiz V.E., Vidal-Melo M.F., Walsh J.L. Strategies for managing oxygenation in obese patients undergoing laparoscopic surgery. Surg Obes Relat Dis. 2015; 11(3): 721-8. DOI: 10.1016/j.soard.2014.11.021

[79] Fernandez-Bustamante A., Wood C.L., Tran Z.V., et al. Intraoperative ventilation: incidence and risk factors for receiving large tidal volumes during general anesthesia. BMC Anesthesiol. 2011; 11: 22. DOI: 10.1186/1471-2253-11-22

[80] Coussa M., Proietti S., Schnyder P., et al. Prevention of atelectasis formation during the induction of general anesthesia in morbidly obese patients. Anesth Analg. 2004; 98(5): 1491-5. DOI: 10.1213/01.ane.0000111743.61132.99

[81] Pelosi P., Ravagnan I., Giurati G., et al. Positive end-expiratory pressure improves respiratory function in obese but not in normal subjects during anesthesia and paralysis. Anesthesiology. 1999; 91(5): 1221-31. DOI: $10.1097 / 00132586-200012000-00043$

[82] Nestler C., Simon P., Petroff D., et al. Individualized positive end-expiratory pressure in obese patients during general anaesthesia: a randomized controlled clinical trial using electrical impedance tomography. Br J Anaesth. 2017 Dec 1; 119(6): 1194-1205. DOI: 10.1093/bja/aex192

[83] Tafer N., Nouette-Gaulain K., Richebé P., et al. Effectiveness of a recruitment manoeuvre and positive end-expiratory pressure on respiratory mechanics during laparoscopic bariatric surgery. Ann Fr Anesth Reanim. 2009; 28(2): 130-4. DOI: 10.1016/j.annfar.2008.12.022

[84] Chalhoub V., Yazigi A., Sleilaty G., et al. Effect of vital capacity manoeuvres on arterial oxygenation in morbidly obese patients undergoing open bariatric surgery. Eur J Anaesthesiol. 2007; 24(3): 283-8. DOI: 10.1017/s0265021506001529

[85] Almarakbi W.A., Fawzi H.M., Alhashemi J.A. Effects of four intraoperative ventilatory strategies on respiratory compliance and gas exchange during laparoscopic gastric banding in obese patients. $\mathrm{Br}$ J Anaesth. 2009; 102(6): 862-8. DOI: 10.1093/bja/aep084

[86] Whalen F.X., Gajic O., Thompson G.B. The effects of the alveolar recruitment maneuver and positive end-expiratory pressure on arterial oxygenation during laparoscopic bariatric surgery. Anesth Analg. 2006; 102: 298-305. DOI: 10.1213/01.ane.0000183655.57275.7a

[87] Talab H.F., Zabani I.A., Abdelrahman H.S., et al. Intraoperative ventilatory strategies for prevention of pulmonary atelectasis in obese patients undergoing laparoscopic bariatric surgery. Anesth Analg. 2009; 109(5): 1511-6. DOI: 10.1213/ane.0b013e3181ba7945

[88] Wei K., Min S., Cao J., et al. Repeated alveolar recruitment maneuvers with and without positive end-expiratory pressure during bariatric surgery: a randomized trial. Minerva Anestesiol. 2018; 84(4): 463-472. DOI: 10.1097/01.aoa.0000530016.91344.81

[89] Writing Committee for the PROBESE Collaborative Group of the PROtective VEntilation Network (PROVEnet) for the Clinical Trial Network of the European Society of Anaesthesiology, Bluth T, Serpa Neto A, Effect of Intraoperative High Positive End-Expira- tory Pressure (PEEP) With Recruitment Maneuvers vs Low PEEP on Postoperative Pulmonary Complications in Obese Patients: A Randomized Clinical Trial. JAMA. 2019; 321(23): 2292-2305. DOI: 10.1001/jama.2019.7505

[90] Ball L., Hemmes S.N.T., Serpa Neto A., Bluth T. Intraoperative ventilation settings and their associations with postoperative pulmonary complications in obese patients. Br J Anaesth. 2018; 121(4): 899-908. DOI: 10.1016/j.bja.2018.04.021

[91] Tusman G., Groisman I., Fiolo F.E., et al. Noninvasive monitoring of lung recruitment maneuvers in morbidly obese patients: The role of pulse oximetry and volumetric capnography. Anesth Analg. 2014; 118: 137-44. DOI: 10.1213/01.ane.0000438350.29240.08

[92] Kim H.J., Kim W.H., Lim H.W., et al. Obesity is independently associated with spinal anesthesia outcomes: a prospective observation study. PLoS One. 2015; 10(4): 0124264. DOI: 10.1371/journal.pone.0124264

[93] Rodrigues F.R., Brandão M.J. Regional anesthesia for cesarean section in obese pregnant women: a retrospective study. Rev Bras Anestesiol. 2011; 61(1): 13-20. DOI: 10.1016/s0034-7094(11)70002-2

[94] Nielsen K.C., Guller U., Steele S.M., et al. Influence of obesity on surgical regional anesthesia in the ambulatory setting: an analysis of 9,038 blocks. Anesthesiology. 2005; 102(1): 181-7. DOI: 10.1097/00000542-200501000-00027

[95] Ikramuddin S., Blackstone R.P., Brancatisano A., et al. Effect of reversible intermittent intra-abdominal vagal nerve blockade on morbid obesity: the ReCharge randomized clinical trial. JAMA. 2014; 312(9): 915-22. DOI: 10.1001/jama.2014.10540

[96] Symeonidis D., Baloyiannis I., Georgopoulou S., et al. Laparoscopic ventral hernia repair in obese patients under spinal anesthesia. Int J Surg. 2013; 11(9): 926-9. DOI: 10.1016/j.ijsu.2013.07.002

[97] Tonidandel A., Booth J., D'Angelo R. Anesthetic and obstetric outcomes in morbidly obese parturients: a 20 -year follow-up retrospective cohort study. Int J Obstet Anesth. 2014; 23(4): 357-64. DOI: 10.1097/01.aoa.0000472733.35350.93

[98] Sudré E.C., de Batista P.R., Castiglia Y.M. Longer Immediate Recovery Time After Anesthesia Increases Risk of Respiratory Complications After Laparotomy for Bariatric Surgery: a Randomized Clinical Trial and a Cohort Study. Obes Surg. 2015; 25(11): 2205-12. DOI: 10.1007/s11695-015-1855-8

[99] Loupec T., Frasca D., Rousseau N., et al. Appropriate dosing of sugammadex to reverse deep rocuronium-induced neuromuscular blockade in morbidly obese patients. Anaesthesia. 2016; 71(3): 265-72. DOI: 10.1111/anae.13344

[100] Llauradó S., Sabaté A., Ferreres E., et al. Postoperative respiratory outcomes in laparoscopic bariatric surgery: comparison of a prospective group of patients whose neuromuscular blockade was reverted with sugammadex and a historical one reverted with neostigmine. Rev Esp Anestesiol Reanim. 2014; 61(10): 565-70. DOI: 10.1016/j.redar.2013.11.009

[101] Monk T.G., Rietbergen H., Woo T., et al. Use of Sugammadex in Patients with Obesity: A Pooled Analysis. Am J Ther. 2017; 24(5): 507-e516. DOI: 10.1016/j.redar.2013.11.009

[102] Carron M., Galzignato C., Godi l., et al. Benefit of sugammadex on lung ventilation evaluated with electrical impedance tomography 
in a morbidly obese patient undergoing bariatric surgery. J Clin Anesth. 2016; 31: 78-9. DOI: 10.1016/j.jclinane.2016.01.018

[103] Castro D.S. Jr., Leão P., Borges S., et al. Sugammadex reduces postoperative pain after laparoscopic bariatric surgery: a randomized trial. Surg Laparosc Endosc Percutan Tech. 2014 Oct; 24(5): 420-3. DOI: $10.1097 /$ sle. 0000000000000049

[104] Mahul M., Jung B., Galia F., et al. Spontaneous breathing trial and post-extubation work of breathing in morbidly obese critically ill patients. Crit Care. 2016; 20(1): 346. DOI: 10.1186/s13054-016-1457-4

[105] Lin H.T., Ting P.C., Chang W.Y., et al. Predictive risk index and prognosis of postoperative reintubation after planned extubation during general anesthesia: a single-center retrospective case-controlled study in Taiwan from 2005 to 2009. Acta Anaesthesiol Taiwan. 2013; 51(1): 3-9. DOI: 10.1016/j.aat.2013.03.004

[106] Parlow J.L., Ahn R., Milne B. Obesity is a risk factor for failure of "fast track" extubation following coronary artery bypass surgery. Can J Anaesth. 2006; 53(3): 288-94. DOI: 10.1007/bf03022217

[107] El-Solh A.A., Aquilina A., Pineda L., et al. Noninvasive ventilation for prevention of post-extubation respiratory failure in obese patients. Eur Respir J. 2006; 28(3): 588-95. DOI: 10.1183/09031936.06.00150705

[108] Nava S., Gregoretti C., Fanfulla F., et al. Noninvasive ventilation to prevent respiratory failure after extubation in highrisk patients. Crit Care Med. 2005 Nov; 33(11): 2465-70. DOI: 10.1097/01.ccm.0000186416.44752.72

[109] Lin C., Yu H., Fan H., et al. The efficacy of noninvasive ventilation in managing postextubation respiratory failure: a meta-analysis. Heart Lung. 2014; 43(2): 99-104. DOI: 10.1016/j.hrtlng.2014.01.002

[110] Ziemann-Gimmel P., Hensel P., Koppman J., et al. Multimodal analgesia reducesnarcotic requirements and antiemetic rescue medication in laparoscopic Roux-en-Y gastricbypass surgery. Surg Obes Relat Dis. 2013; 9(6): 975-80. DOI: 10.1016/j.soard.2013.02.003

[111] Song K., Melroy M.J., Whipple O.C. Optimizing multimodal analgesia with intravenousacetaminophen and opioids in postoperative bariatric patients. Pharmacotherapy. 2014; 34(1): 14-21. DOI: 10.1002/phar.1517

[112] Madan A.K., Ternovits C.A., Speck K.E., et al. Inpatient pain medicationrequirements after laparoscopic gastric bypass. Obes Surg. 2005; 15(6): 778-81. DOI: 10.1381/0960892054222812

[113] Karlnosk R.A., Sprenker C., Puri S., et al. Reduced postoperative pain and complications after a modified multidisciplinary approach for bariatric surgery. Open Obes J. 2013; 5: 60-4. DOI: 10.2174/1876823720130508009

[114] Эпштейн С.Л. Периоперационное анестезиологическое обеспечение больных с морбидным ожирением. Регионарная анестезия и лечение острой боли. 2012. 6(3): 5-27. DOI: 10.21518/2079-701X-2013-5-6-17-27 [Epshteyn S.L. Perioperatsionnoye anesteziologicheskoye obespecheniye bolnykh s morbidnym ozhireniyem. Regionarnaya anesteziya i lecheniye ostroy boli. 2012. 6(3): 5-27. (In Russ)]

[115] Zotou A., Siampalioti A., Tagari P., et al. Does epiduralmorphine loading in addition to thoracic epidural analgesia benefit the postoperative management of morbidly obese patients undergoing open bariatric surgery? A pilot study. Obes Surg. 2014; 24(12): 2099-108. DOI: 10.1007/s11695-014-1305-z
[116] Schumann R., Shikora S., Weiss J.M., et al. A comparison of multimodal perioperative analgesia to epidural pain management after gastric bypass surgery. Anesth Analg. 2003; 96(2): 469-74. DOI: 10.1213/00000539-200302000-00032

[117] Michaloudis D., Fraidakis O., Petrou A., et al. Continuous spinal anesthesia/analgesia for perioperative management of morbidly obese patients undergoing laparotomy for gastroplastic surgery. Obes Surg. 2000; 10(3): 220-9. DOI: 10.1381/096089200321643494

[118] Nielsen K.C., Guller U., Steele S.M., et al. Influence of obesity on surgical regional anesthesia in the ambulatory setting: an analysis of 9,038 blocks. Anesthesiology. 2005; 102(1): 181-7. DOI: 10.1097/00000542-200501000-00027

[119] Franco C.D., Gloss F.J., Voronov G., et al. Supraclavicular block in the obese population: an analysis of 2020 blocks. Anesth Analg. 2006; 102(4): 1252-4. DOI: 10.1213/01.ane.0000198341.53062.a2

[120] Schroeder K., Andrei A.C., Furlong M.J., et al. The perioperative effect of increased body mass index on peripheral nerve blockade: an analysis of 528 ultrasound guided interscalene blocks. Rev Bras Anestesiol. 2012; 62(1): 28-38. DOI: 10.1016/s0034-7094(12)70100-9

[121] Naja Z.M., Naccache N., Ziade F., et al. Multilevel nerve stimulator guided paravertebral block as a sole anesthetic technique for breast cancer surgery in morbidly obese patients. J Anesth. 2011; 25(5): 760-4. DOI: 10.1007/s00540-011-1194-4

[122] Brodsky J.B., Mariano E.R. Regional anaesthesia in the obese patient: lost landmarks and evolving ultrasound guidance. Best Pract Res Clin Anaesthesiol. 2011; 25(1): 61-72. DOI: 10.1016/j.bpa.2010.12.005

[123] Rottenstreich A., Levin G., Elchalal U., et al. The effect on thrombin generation and anti-Xa levels of thromboprophylaxis dose adjustment in post-cesarean obese patients - A prospective cohort study. Thromb Res. 2018; 170: 69-74. DOI: 10.1016/j.thromres.2018.08.007

[124] Rottenstreich A., Elazary R., Yuval J.B., et al. Assessment of the procoagulant potential after laparoscopic sleeve gastrectomy: a potential role for extended thromboprophylaxis. Surg Obes Relat Dis. 2018; 14(1): 1-7. DOI: 10.1016/j.soard.2017.09.526

[125] Moaad F., Zakhar B., Anton K., et al. Is LMWH Sufficient for Anticoagulant Prophylaxis in Bariatric Surgery? Prospective Study. Obes Surg. 2017; 27(9): 2331-2337. DOI: 10.1007/s11695-017-2638-1

[126] Moulin P.A., Dutour A., Ancel P., et al. Perioperative thromboprophylaxis in severely obese patients undergoing bariatric surgery: insights from a French national survey. Surg Obes Relat Dis. 2017 Feb; 13(2): 320-326. DOI: 10.1016/j.soard.2016.08.497

[127] Steib A., Degirmenci S.E., Junke E., et al. Once versus twice daily injection of enoxaparin for thromboprophylaxis in bariatric surgery: effects on antifactor Xa activity and procoagulant microparticles. A randomized controlled study. Surg Obes Relat Dis. 2016; 12(3): 613-621. DOI: 10.1016/j.soard.2015.08.505

[128] Magee C.J., Barry J., Javed S., et al. Extended thromboprophylaxis reduces incidence of postoperative venous thromboembolism in laparoscopic bariatric surgery. Surg Obes Relat Dis. 2010; 6(3): 322-5. DOI: 10.1016/j.soard.2010.02.046

[129] Rocha A., et al. Risk of venous thromboembolism and efficacy of thromboprophylaxis in hospitalized obese medical patients and in obese patients undergoing bariatric surgery. Obes Surg. 2006; 16(12): 1645-1655. DOI: 10.1381/096089206779319383 